\title{
Evidence for an attentional component in saccadic inhibition of return
}

\author{
David Souto $\cdot$ Dirk Kerzel
}

Published online: 27 June 2009

(c) Springer-Verlag 2009

\section{Erratum to: Exp Brain Res (2009) 195:531-540 DOI 10.1007/s00221-009-1824-3}

There was a mistake when referring to invalid and valid conditions. In Table 1 we inverted the "invalid" and "valid" labels. In contrast to what is shown in Table 1, reaction times (RTs) were always longer in the valid condition. In the main text, IOR was defined as RT in valid trials minus RT in invalid trials, and therefore positive values were obtained. In the legends of Table 1, Figs. 2 and 3, we erroneously stated that IOR was defined as "invalid RT minus valid RT", which should lead to negative values. Finally, when referring to the RT distributions shown in Fig. 2c, the valid trials distribution is shifted rightward and not leftward. We would like to thank Anna Montagnini for pointing out these mistakes to us.
The online version of the original article can be found under doi: $10.1007 / \mathrm{s} 00221-009-1824-3$.

D. Souto $(\bowtie) \cdot$ D. Kerzel

Faculté de Psychologie et Sciences de l'Éducation,

Université de Genève, 40 bd du Pont d'Arve,

1205 Geneva, Switzerland

e-mail: david.souto@unige.ch
The correct Table 1 is given below:

Table 1 Mean reaction time (RT) and between-subject standard error of the mean in Experiments 1 and 2 in the format $M \pm$ SE. Inhibition of return (IOR) is the difference between valid and invalid conditions

\begin{tabular}{|c|c|c|c|c|c|c|}
\hline & \multicolumn{6}{|c|}{ Experiment $1(N=22)$} \\
\hline & \multicolumn{3}{|c|}{ Saccadic RT (ms) } & \multicolumn{3}{|c|}{ Manual RT (ms) } \\
\hline & Valid & Invalid & IOR & Valid & Invalid & IOR \\
\hline \multicolumn{7}{|l|}{ Gap } \\
\hline High contrast & $178 \pm 6$ & $155 \pm 6$ & 23 & $319 \pm 9$ & $298 \pm 8$ & 21 \\
\hline Low contrast & $228 \pm 10$ & $187 \pm 7$ & 41 & $365 \pm 10$ & $336 \pm 9$ & 29 \\
\hline \multicolumn{7}{|l|}{ Overlap } \\
\hline High contrast & $231 \pm 8$ & $207 \pm 6$ & 24 & $325 \pm 10$ & $312 \pm 8$ & 13 \\
\hline \multirow[t]{4}{*}{ Low contrast } & $274 \pm 9$ & $241 \pm 8$ & 33 & $372 \pm 10$ & $349 \pm 9$ & 23 \\
\hline & \multicolumn{6}{|c|}{ Experiment $2(N=18$, saccadic responses $)$} \\
\hline & \multicolumn{3}{|c|}{ Light background } & \multicolumn{3}{|c|}{ Dark background } \\
\hline & Valid & Invalid & IOR & Valid & Invalid & IOR \\
\hline \multicolumn{7}{|l|}{ Step } \\
\hline High contrast & $212 \pm 9$ & $189 \pm 8$ & 23 & $203 \pm 8$ & $181 \pm 5$ & 22 \\
\hline Low contrast & $258 \pm 10$ & $211 \pm 8$ & 47 & $211 \pm 8$ & $192 \pm 6$ & 19 \\
\hline \multicolumn{7}{|l|}{ Overlap } \\
\hline High contrast & $246 \pm 12$ & $204 \pm 8$ & 42 & $237 \pm 12$ & $206 \pm 9$ & 31 \\
\hline Low contrast & $305 \pm 15$ & $262 \pm 11$ & 43 & $276 \pm 14$ & $219 \pm 9$ & 57 \\
\hline
\end{tabular}

